\title{
Exploiting X-ray induced anisotropic lattice changes to improve intensity extraction in protein powder diffraction: Application to heavy atom detection
}

\section{Besnard ${ }^{1 *}$, F. Camus ${ }^{1}$, M. Fleurant ${ }^{1}$, A. Dahlström ${ }^{1}$, J. P. Wright ${ }^{2}$, I. Margiolaki ${ }^{2}$, P. Pattison ${ }^{1,3}$, M. Schiltz ${ }^{1}$}

${ }^{1}$ Ecole Polytechnique Fédérale de Lausanne (EPFL), Laboratoire de Cristallographie, Switzerland

${ }^{2}$ European Synchrotron Radiation Facility (ESRF), Grenoble, France

${ }^{3}$ Swiss-Norwegian Beamlines, E.S.R.F. Grenoble, France

* Contact author; e-mail: celine.besnard@epfl.ch

Keywords: powder diffraction, protein crystallography, radiation damage, synchrotron radiation.

\begin{abstract}
X-ray induced anisotropic variations of cell parameters in porcine pancreatic elastase (PPE) were used in a multi-Pawley refinement in order to improve the deconvolution of overlapping peaks occurring in the high-angle region of the powder pattern. The benefit of combining scans is demonstrated by an improvement in the quality of the isomorphous difference Patterson maps used to detect the positions of heavy atoms in a uranyl derivative of PPE.
\end{abstract}

\section{Introduction}

During the last decade, substantial progress has been achieved in applying the powder diffraction technique to protein samples. If the recent developments in structure refinement, molecular replacement and ligand detection have shown the applicability of the method [1] extracting a good set of integrated intensities from a protein powder diffraction pattern remains a non-trivial problem. The collapse of the three dimensional diffraction data onto the one dimension of a powder diffraction pattern inevitably leads to peak overlap. With protein samples, the large unit-cell parameters further aggravate this problem. As a consequence, there is a loss of information, particularly at short d-spacings, and the extraction of reliable structure-factor amplitudes from powder data becomes challenging. One method to partially overcome this problem consists in exploiting anisotropic shifts in the unit cell parameters. A set of patterns is recorded in which the variation of a physical parameter produces an anisot- 
ropic change in the unit-cell parameters, without disturbing too much the crystal structure. Due to the anisotropy of the lattice changes, neighbouring peaks that are accidentally overlapped in one pattern may be well-enough separated in another one, so that their individual reflection intensities can be determined. Thus, by combining the information contained in a series of patterns, an improvement of the extracted intensities in the region where overlap occurs is possible. This method has been successfully applied by making use of anisotropic thermal expansion [2] or variations in the $\mathrm{pH}$ of the crystallization buffer [3].

Radiation damage caused by the exposure of samples to an intense X-ray beam is a welldiscussed phenomenon in single-crystal protein crystallography, since it can seriously hamper structure determination [4]. Upon exposure to X-rays, the diffraction power is progressively lost, especially at high angles. Another effect that appears to be universal is an irreversible radiation-induced lattice expansion. In many cases the lattice expansion is anisotropic. However, the mechanism by which the unit-cell expands is not understood. In order to prevent radiation damage, cryo-cooling is usually employed in single-crystal studies. Despite some recent progress [5], it remains difficult to cool protein powder samples without compromising the quality of their diffraction profile. Therefore, most protein powder diffraction experiments are carried out at room temperature, which generally results in observable radiation damage. Advantage can be taken of the anisotropic peak shifts associated with radiationinduced processes in order to help in deconvoluting overlapping peaks, provided that the structural changes produced by the X-rays remain at a tolerable level.

In the present study, we have applied this approach to the problem of detecting heavy atoms in derivative protein crystals. The method of isomorphous replacement is a well-established technique for solving protein structures from single-crystal data. The method exploits the differences in measured intensities between a native sample and a heavy atom derivative. The first step of this method consists in detecting the position of the heavy atoms by Patterson maps computed from intensity differences. We have used the protein PPE, of which a well-characterized uranyl derivative can be prepared, as a test case for evaluating the improvements in data quality that can be achieved by combining several powder patterns that are affected by radiation-induced anisotropic lattice changes.

\section{Experimental part}

\section{Sample preparation}

Two polycrystalline samples were prepared: a native PPE sample and a uranyl derivative obtained by soaking for a few days the native protein in a solution of uranyl nitrate (Concentration of about $5 \mathrm{mM}$ ). Both samples were well crystallized and were ground prior to the diffraction experiment. Glass capillaries were filled with the samples and centrifuged. Excess mother liquor was taken out before sealing off the capillaries with wax.

\section{Data collection}

A series of angular scans were collected on the high resolution powder diffraction beamline BM01B at the Swiss-Norwegian Beamline at the ESRF, Grenoble. The beamline is equipped with a set of six Si (111) analyser crystals, providing a good angular resolution with an instrumental peak broadening of about $0.01^{\circ}$. The average FWHM observed on the collected pattern is less than $0.02^{\circ}$. The beamline is installed on an ESRF bending magnet source. An estimate of the flux on the sample at the wavelength of $0.8 \AA$ used to collect the data is $10^{10}$ 
photon/sec. This reasonable flux allowed a full series of patterns to be recorded before the crystals were seriously radiation-damaged. A speed of $2 \mathrm{~s} / \mathrm{step}$, and steps of $0.004^{\circ}$ were used. All the measurements were carried out at room temperature. Under those conditions, 40 patterns were collected before the native PPE crystals were destroyed (range $0.3^{\circ}-12^{\circ}$ ) and 15 patterns were recorded on its uranyl derivative (range $0.3^{\circ}-10^{\circ}$ ).

\section{Intensity extraction}

The intensity extraction was carried out using the TOPAS ACADEMIC [6] software. In a first step, a LeBail fit was carried out on each individual scan, in which profile parameters, background and unit-cell parameters were refined. Then, all the patterns were combined together in a Pawley refinement. In this refinement, a single set of intensities was extracted from all the patterns of the same sample, this being possible in the TOPAS software implementation by using hard constraints on the intensities. For each pattern, a constant scale parameter was also refined in order to take care of the overall loss of intensity due to the radiation damage. More sophisticated scaling models using isotropic scale parameters were also tested, but without discernable improvement.

\section{Patterson map calculation}

The extracted intensities were converted to structure factor amplitudes after the application of Lorentz-polarization corrections. This list of structure factor amplitudes was introduced into the CCP4 program suite [7], in which all further calculations were done. The data of the uranyl derivative were scaled to the native data by using an anisotropic scaling model, after which an origin-removed isomorphous-difference Patterson map was calculated.

\section{Results and discussion}

The normalized unit cell parameters have been plotted in figure 1(a) as a function of the exposure time to X-rays. The changes in the crystal lattice are clearly anisotropic, with a much larger variation for the $a$-parameter. After substantial irradiation, the behaviour of the unit cell parameters is changing abruptly from an expansion to a contraction. As can been seen from the increase in the error bars, the unit cell parameters are much less welldetermined in this high-exposure region. This is due to a global deterioration of the quality of the diffraction pattern, as can be seen by comparing the first scan to the $25^{\text {th }}$ scan (figure 2) for the native PPE. This loss of diffraction intensity is reflected in the scaling parameters refined in the fitting procedure which are also shown in figure 2.

There is a slight difference between the behaviour of native PPE and its uranyl derivative. In the derivative compound, the changes in the unit-cell parameters seem to be less rapid for an equivalent loss of diffracting power. There have been many discussions about unit-cell variations caused by radiation damage [8] but no satisfactory theory is yet available. However, for the purposes of this study, the important point is the strongly anisotropic change in the unitcell parameters and the rather moderate loss in intensities at the beginning of the radiationdamage process. These factors allowed the combination of the observed powder patterns in a multi-Pawley fitting procedure. 

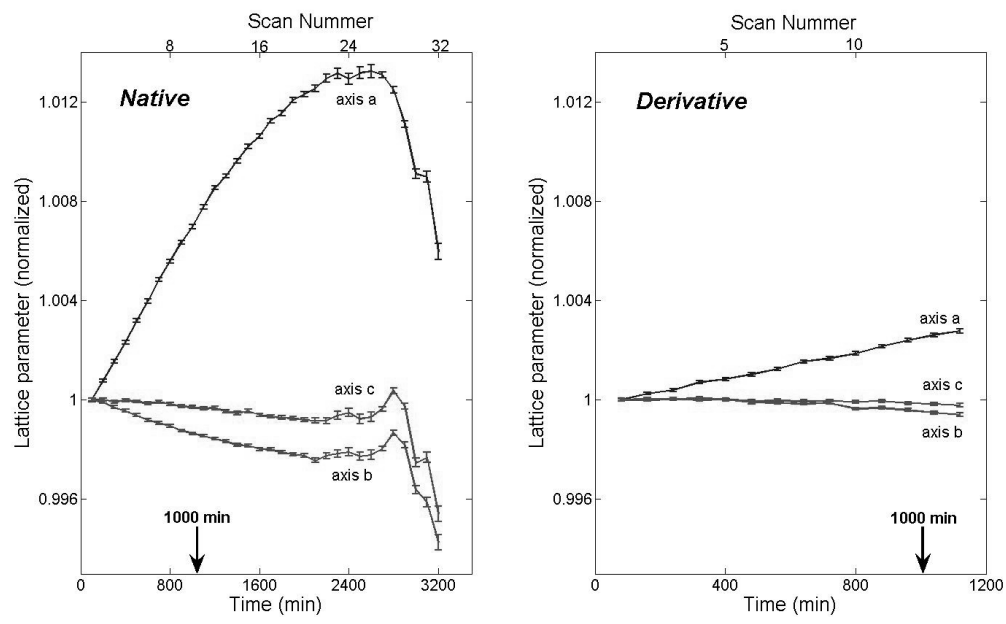

Figure 1. Variation of the lattice parameters for native PPE (left) and its uranyl derivative (right) as a function of the irradiation time. PPE crystallizes in the orthorhombic space-group $P 2_{1} 2_{1} 2_{1}$ with unit cell parameters 51.48357 .99175 .29 (A). The lattice parameters have been normalized to the first scan parameters in order to ease the comparison between the three axes. Both graphs are on the same vertical scale.
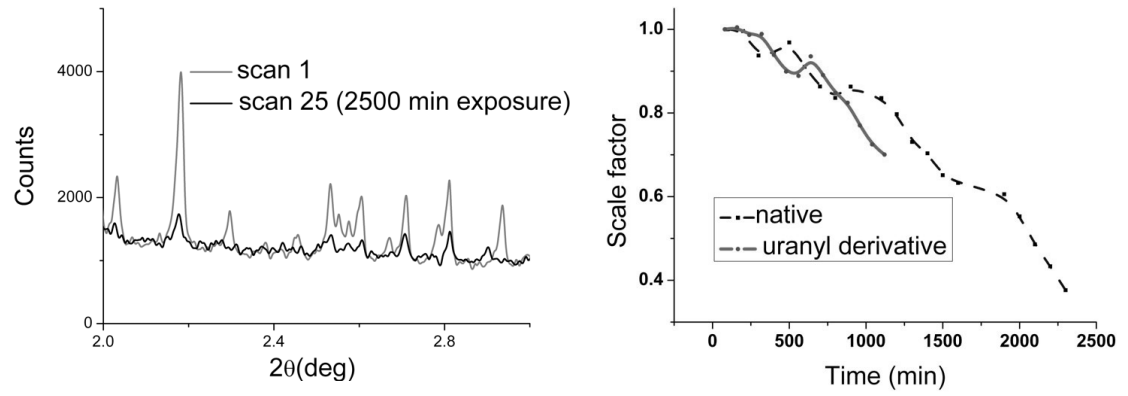

Figure 2. Comparison of the diffraction scans for native PPE at the beginning of the experiment and after 25 scans (left). The deterioration of the quality of the data is clearly visible. The intensity loss is reflected in the scale factors refined for each pattern which are plotted for uranylated and native PPE as a function of the time (right).

In order to test the improvement in the extracted intensities due to the combination of patterns, different combined Pawley refinements have been carried out using more and more patterns. The extracted intensities are expected to improve as more patterns are added. Indeed, the effect on the isomorphous difference Patterson maps calculated from these ex- 
tracted intensities is dramatic. Starting with 5 patterns, the Harker peaks do not appear clearly in the map. In figure 3 are shown the isomorphous difference Patterson maps using respectively 20, 25 and 29 scans for native PPE and 8 scans for uranylated PPE. The peaks appear most clearly in the map where 25 scans of native data have been combined, whereas they are much less prominent in the other maps. This illustrates the competition between the gain obtained by the anisotropic shift of the lattice parameters (improvement in going from 20 to 25 patterns) and the loss of data quality associated to radiation damage (deterioration in going from 25 to 29 patterns).
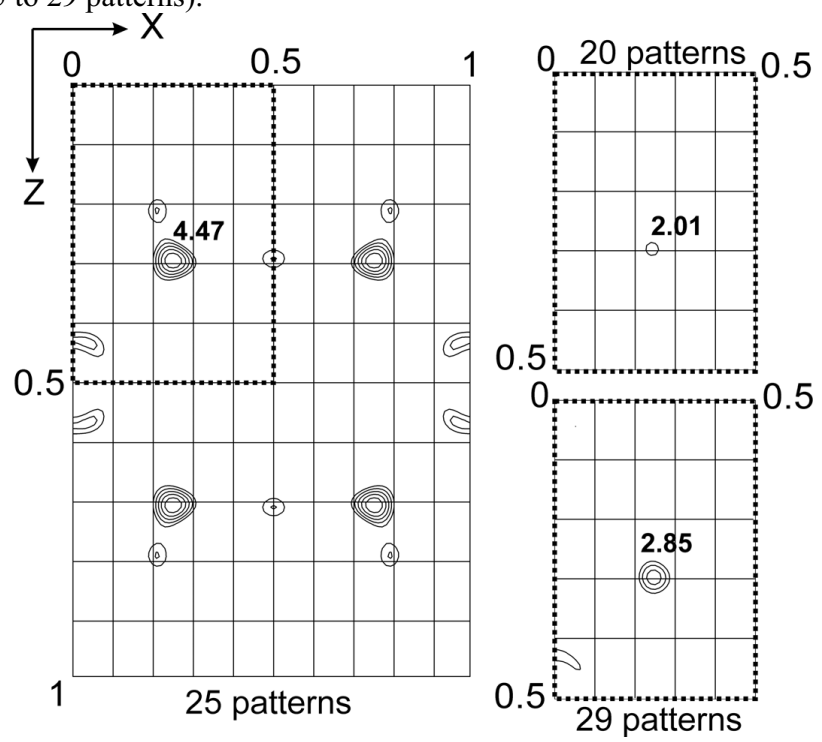

Figure 3. Isomorphous difference Patterson maps obtained by combining respectively 25, 20 and 29 scans of native PPE during the multi-Pawley fitting procedure. For all the maps, 8 scans were combined in the intensity extraction for the uranyl derivative. The height of the Patterson peak $(I / \sigma)$ is given for each map. The contours used start at $1.5 \sigma$ with a step of 0.5 .

Table 1 presents the $I / \sigma$ observed for the heavy-atom Harker peaks shown in the maps of figure 3 as a function of the number of patterns combined for both the uranylated and the native samples. There appears to be an optimum in the combination of 8 patterns for the uranyl derivative and 25 patterns for the native. Including more scans for either the native or the derivative data leads to a deterioration of the signal, most likely because the intensities change as a result of severe radiation damage. The good results obtained for the combination of respectively 29 scans of the native and 11 scans of the derivative may indicate a similar evolution of intensity changes due to radiation damage in both samples. Thus, by computing maps from intensity differences, the radiation-induced intensity changes would partially cancel out. 
Table 1. Maximum (I/ $\sigma)$ of the peak observed in the Isomorphous Difference Patterson map at the expected position 0.240 .50 .29 as a function of the number of scans combined in the multi-Pawley refinement for the native and derivative PPE. The Patterson map was calculated with a resolution cutoff at 6 A. The symbol * indicates that the peak has an $I / \sigma$ value less than 1.5 .

Added scans for native

\begin{tabular}{|c|c|c|c|c|c|c|}
\hline & & 25 & 20 & 25 & 29 & 34 \\
\hline Added & 3 & $*$ & $*$ & $*$ & $*$ & $*$ \\
\hline scans for & 8 & $*$ & 2.01 & 4.47 & 2.85 & $*$ \\
\hline \multirow[t]{2}{*}{ derivative } & 11 & $*$ & $*$ & * & 4.66 & $*$ \\
\hline & 14 & $*$ & $*$ & $*$ & 2.92 & $*$ \\
\hline
\end{tabular}

\section{Conclusion}

$\mathrm{X}$-ray induced processes in crystalline protein samples can give rise to anisotropic changes in the lattice parameters. These changes can be exploited to help the extraction of better integrated intensities from a series of powder diffraction patterns. This improvement has been demonstrated in the detection of heavy atoms in isomorphous difference Patterson maps computed from data collected on a native and a uranyl derivative of PPE. There is a clear improvement in the maps when a series of patterns affected by radiation-induced lattice changes are combined in the intensity extraction process. However, since radiation damage is a destructive process, care has to be taken to retain a moderate dose so that non-isomorphism between the different combined patterns is kept at a minimum level.

\section{References}

1. Von Dreele, B., 2003, Methods Enzymol., 368, 254-267.

2. Shankland, K., David, W. I. F. \& Sivia, D. S., 1997, J. Mater. Chem., 7, 569-572.

3. Basso, S., Fitch, A. N., Fox, G. C., Margiolaki, I., Wright, J. P., 2005, Acta Cryst. $D, \mathbf{6 1}, 1612-1625$.

4. Nave, C. \& Garman, E. F., 2005, J. Synchrotron Rad., 12, 257-260.

5. Jenner M. J., Wright J. P., Margiolaki I., Fitch, A. N., 2006, to be published

6. Coelho, A., 2004, TOPAS-Academic. http://members.optusnet.com.au/ alancoelho/

7. Collaborative Computation Project, Number 4, 1994, Acta Cryst D, 50, 760-763; http://www.dl.ac.uk/CCP/CCP4

8. Ravelli, R. B. G., Theveneau, P., McSweeney, S. \& Caffrey, M., 2002, J. Synchrotron Rad., 9, 355-360. 